Resenhas / Reviews

\title{
The human right to health
}

\author{
Jonathan Wolff, 1. ed. New York: W. W. Norton \& \\ Company, 2013, Norton Global Ethics Series
}

Resenha por: Lucas de Moura Rodrigues*

\section{Introdução}

O autor é, atualmente, professor de filosofia e diretor do Centre for Philosophy, Justice and Health na University College London, bem como membro do Nuffield Council on Bioethics e do Nuffield Council Working Party on Personalised Healthcare. Já participou de organizações como Nuffield Council Working Party on the Ethics of Research Involving Animals, Academy of Medical Sciences, Working Party on Brain Science and Addiction, British Philosophical Association e Aristotelian Society. Notadamente, um histórico não apenas no âmbito acadêmico, mas também no âmbito político-institucional. Essa experiência se expressa sensivelmente em suas obras, seja na forma da apresentação das ideias, seja no tema.

The Human Right to Health insere-se na discussão sobre se a saúde deve ser instituída como um direito humano. Diante das complexas questões teóricas e práticas envolvidas no reconhecimento da saúde, alguns argumentam contrariamente a isso, afirmando que ela deve ser tratada de maneira mais pragmática ("in a more pragmatic fashion"), ${ }^{(1)}$ numa relação de custo-benefício, enquanto outros, mais idealistas ("more idealistic")(2) diante dos graves problemas de saúde no mundo, entendem que, mesmo com as complexas questões teóricas e práticas, a saúde deve ser tratada como uma questão jurídica.

O autor define seu posicionamento como um idealismo moderado: "this book will be an exercise in cautious idealism", ${ }^{(3)}$ isto é, entende que os problemas de

\footnotetext{
* Graduando, Faculdade de Direito, Universidade de São Paulo; Orientando de iniciação científica, Núcleo de Pesquisas em Direito Sanitário da Universidade de São Paulo. São Paulo/SP - Brasil. E-mail: lucas.moura.rodrigues@usp.br

Resenha recebida em: 06/01/2014. Aprovada em: 08/01/2014.

1 WOLFF, Jonathan. The human right to health. Nova York: W. W. Norton \& Company, 2012. p. XV.

2 Id. Ibid. p. XV.

${ }^{3}$ Id., loc. cit.
} 
saúde global não devem ser tratados numa mera relação de custo-benefício, e sim como uma questão jurídica. No entanto, pondera que o reconhecimento de seu status jurídico deve vir acompanhado de um exercício de problematização em vista das questões teóricas e, sobretudo, práticas relacionadas à saúde, no sentido de definir os limites do direito humano à saúde em contornos realistas, possíveis, praticáveis. É à demonstração de que é possível e imperativo tratar a saúde como um direito humano, mesmo diante das dificuldades existentes, que se dedica ao longo de todo o livro.

\section{Ideias gerais}

Dedutivamente, uma demonstração desse tipo exige que o autor enfrente as referidas dificuldades relacionadas ao reconhecimento do status jurídico da saúde. Parece ser consenso que, havendo uma situação problemática (por exemplo, relacionada com a saúde), existe um dever moral, inerente ao ser humano, de empenhar esforços no sentido de solucioná-la. No entanto, como foi dito, não é consenso que esse dever seja tratado como uma questão jurídica. A fim de esclarecer a possibilidade e mesmo imperatividade disso, o autor dedica-se às seguintes questões: em que consiste esse dever moral - "but what sort of duty is this? And conversely, what sorts of moral claims does each individual have to a full life in good health?"(4) - e o que significa existir um direito à saúde e, se, de fato, existe, a que dá direito - "is there really a right to health? What does this actually mean? What does it call for in practice?". ${ }^{(5)}$

Se um problema é gerado, perpetuado de maneira sistemática, isto é, em função de uma série de decisões internas e externas que conformam circunstâncias problemáticas - "a set of other people's decisions - decisions about the pricing of drugs, patent laws, economic policy, national priorities, and international sanctions. (...). Paul Farmer calls this 'structural violence'"(6) -, e se aquele problema pode ser, de fato, solucionado - "the Universal Declaration has its origins (...) in what apparently civilized people were capable of doing to one another and to their own citizens"(7) -, parece ser consenso que existe o dever moral de empenhar esforços no sentido de solucioná-lo. Nesse sentido, países que apresentem condições e serviços de saúde (atendimento médico, medicamentos, equipamentos, informações) mais eficazes, eficientes e acessíveis ou organismos que detenham direito de exclusividade sobre a reprodução de determinado bem que contribua com a melhoria da saúde (propriedade intelectual) têm o dever moral de cooperar com a melhoria das condições e dos serviços em outros países.

\footnotetext{
${ }^{4}$ WOLFF, Jonathan. op. cit. p. XIV.

${ }^{5}$ Id., loc. cit.

${ }^{6}$ Id. Ibid., p. 2.

7 Id. Ibid., p. 4.
} 
Evidentemente, este dever moral não se limita a oferecer tratamento médico no sentido de solucionar eventuais surtos de doenças. Com base na mensagem do presidente Truman, na Conferência Internacional de Saúde, 1946, Nova lorque, esse era o entendimento predominante no contexto de inauguração da Organização Mundial da Saúde, o órgão competente para coordenar as ações, bem como para deliberar sobre assuntos relacionados à saúde:

Modern transportation has made it impossible for a nation to protect itself against the introduction of disease by quarantine. This makes it necessary to develop strong health services in every country, which must be coordinated through internactional action. ${ }^{(8)}$

Atualmente, esse direito vai além do oferecimento de tratamento médico. Como atestam os atos normativos que disciplinam o direito à saúde (especificamente, artigo 12(1), Pacto Internacional de Direitos Econômicos, Sociais e Culturais $\left[\right.$ PIDESC] ${ }^{(9)}$ e artigo XXV, Declaração Universal dos Direitos Humanos), ${ }^{(10)}$ ele se estende a todos os seus determinantes (sendo o oferecimento de tratamento médico apenas um deles).

Também evidente, a garantia dos diversos determinantes da saúde deve passar por vários aspectos. Com base no Comentário Geral 14 referente ao artigo $12(2),{ }^{(11)}$ a garantia, ao contrário do que se pode pensar, não deve contemplar apenas disponibilidade (colocar serviços à disposição da população, oferecê-los, na medida de suas necessidades), mas também acessibilidade (garantir os acessos físico e econômico aos serviços e às informações, de maneira não discriminatória), admissibilidade (garantir a aceitação dos serviços, tendo em vista valores éticos próprios da medicina, bem como valores morais, p.ex., próprios de uma minoria, relacionados a gênero, à época da vida etc.) e qualidade dos serviços (garantir a qualificação dos profissionais da saúde, bem como o funcionamento das instalações, dos equipamentos, a validade dos medicamentos etc.).

${ }^{8}$ WOLFF, Jonathan. op. cit., p. 5.

9 Art. 12(1): "Os Estados Partes do presente Pacto reconhecem o direito de toda pessoa desfrutar o mais elevado nível possível de saúde física e mental”. BRASIL. Procuradoria Regional da República da Quarta Região. Pacto Internacional de Direitos Econômicos, Sociais e Culturais. Disponível em: <http://www.prr4.mpf.gov.br/pesquisaPauloLeivas/arquivos/PIDESC.pdf>. Acesso em: 19 jan. 2014.

${ }^{10}$ Art. XXV "Toda pessoa tem direito a um padrão de vida capaz de assegurar a si e a sua família saúde e bem estar, inclusive alimentação, vestuário, habitação, cuidados médicos e os serviços sociais indispensáveis, e direito à segurança em caso de desemprego, doença, invalidez, viuvez, velhice ou outros casos de perda dos meios de subsistência fora de seu controle". BRASIL. Ministério da Justiça. Declaração Internacional dos Direitos Humanos. Disponível em: http://portal. mj.gov.br/sedh/ct/legis_intern/ddh_bib_inter_universal.htm Acesso em: 19 jan. 2014.

${ }^{11}$ Art. 12(2): "As medidas que os Estados partes do presente Pacto deverão adotar com o fim de assegurar o pleno exercício desse direito incluirão as medidas que se façam necessárias para assegurar: a) a diminuição da mortalidade infantil, bem como o desenvolvimento são das crianças; b) a melhoria de todos os aspectos de higiene do trabalho e do meio ambiente; c) a prevenção e tratamento das doenças epidêmicas, endêmicas, profissionais e outras, bem como a luta contra essas doenças; d) a criação de condições que assegurem a todos assistência médica e serviços médicos em caso de enfermidade". BRASIL. Procuradoria Regional da República da Quarta Região. Pacto Internacional de Direitos Econômicos, Sociais e Culturais, cit. 
Aliás, saliente-se que o direito à saúde é entendido como um direito que envolve essencialmente mais do que uma não interferência ("direitos civis e políticos"), mas também uma ação ("direitos econômicos, sociais e culturais"). Em que pese essa dicotomia tenha sido, em grande parte, desmistificada, tendo em vista que tanto uma categoria de direitos quanto a outra exige tanto abstenção (por parte do Estado) quanto proteção (contra a violação por terceiros) e ação (no sentido de promover o direito) ${ }^{(12)}$, não se pode ignorar a resistência que persiste contra direitos econômicos, sociais e culturais, de acordo com o autor, provavelmente apoiada sobre razões ideológicas ${ }^{(13)}$. E o direito à saúde está compreendido na categoria dos direitos econômicos, sociais e culturais, cuja ratificação (Pidesc) foi bastante controversa.

Diante de todas essas dificuldades relacionadas à institucionalização da saúde (com destaque para a falta de recursos suficientes para concretizar o direito), o Comitê do PIDESC instituiu o Comentário Geral 14. Esse entendimento esclarece que o PIDESC não exige a realização total imediata ("full immediate realization") ${ }^{(14)}$ do direito à saúde, mas sim uma realização continuada e progressiva ("progressive realization"), ${ }^{(15)}$ de modo que a violação do direito possa ser justificada, desde que, pelo menos, o país demonstre já ter tomado providências no sentido de corrigir a situação.

No entanto, esse entendimento mal soluciona o problema. De maneira aparentemente contraditória em relação ao Comentário apresentado, o Comentário Geral 3 determina que os países devem empregar todo e qualquer recurso no sentido de garantir a "atenção básica à saúde" ("primary health care"), ${ }^{(16)}$ de modo a sugerir um conteúdo mínimo ("minimum core obligations") (17) cujo descumprimento não pode ser escusado. ${ }^{(18)} \mathrm{O}$ autor apresenta uma série de questões: que dizer dos países que sequer apresentam recursos para garantir a atenção básica à saúde? Se não são capazes de cumpri-lo, qual seria a utilidade de exigir-lhes isso? Seria esse o caso de buscar ajuda internacional?

Aliás, saliente-se que, embora nem todos os países tenham ratificado os atos normativos que disciplinam o direito à saúde (com destaque para PIDESC), este seria exigível por todos e contra todos:

What, though, should we say about countries that had not ratified the Covenant on Civil and Political Rights? (...). (...) we are likely

\footnotetext{
12 "The argument, however, that first-generation rights are cheap and easy to enforce, and secondgeneration rights prohibitively expensive, has now been thoroughly discredited. (...). For example, (...) rights to liberty and security. The state has the a duty not to undermine our liberty and security, but also to protect us from violations of these rights by other citizens. Such protection requires criminal and civil justice systems with vast resources devoted to the police, the law courts, and prisons (...)". WOLFF, Jonathan. op. cit. p. 14.

${ }^{13}$ Id. Ibid., p. 14.

${ }^{14}$ Id. Ibid., p. 10.

${ }^{15}$ Id., loc. cit.

${ }^{16}$ Id. Ibid., p. 11.

${ }^{17}$ Id., loc. cit.

${ }^{18}$ Id., loc. cit.
} 
to believe that such rights now form part of what can be called 'international customary law': morally and legally binding on all countries once there is significant international weight behind them, whatever an individual's state attitude. ${ }^{(19)}$

Afinal, essa seria a diferença essencial dos direitos humanos em relação a outras espécies de direitos, de modo que, reconhecido um direito humano, qualquer indivíduo teria o direito de exigir determinada conduta do país de origem (mesmo que este não tenha ratificado os atos normativos internacionais). Caso o país não o cumprir satisfatoriamente, a pessoa poderia reivindicar isso em nível internacional, e as respostas variariam desde inspeção até intervenção militar:

Essentially human rights have a double role. On the one hand, they provide a statement of the minimum moral obligations owed to human beings simply by virtue of their existence as human beings. On the other, they generate a mechanism of accountability beyond the nation-state. (...). To enshrine something as a human right is to open up internal affairs of a country to international scrutinity. (...): 'I can agitate against my government, and if she does not fulfill her pledge, I shall have and feel the moral support of the whole world'.(20)

No entanto, não se pode ignorar a resistência à ideia de os países estarem obrigados a financiar problemas de saúde do mundo todo. É verdade que já existem alternativas criativas no sentido de tornar isso mais viável, como a criação de fundos internacionais a fim de retribuir contribuições de países e de organismos internacionais no sentido de melhorar as condições de saúde em outros países; no entanto, essa é uma iniciativa ainda incipiente.

O autor questiona: diante desses problemas todos, por que insistir no reconhecimento do direito à saúde? - "Given these problems, insisting on a right to health looks like a hard road to travel. Why attempt it?". (21) Afinal, como dito a princípio, já existe um dever moral de ajudar pessoas em situações problemáticas, fruto de um senso altruísta inerente ao ser humano.

O autor argumenta: não é que, se a saúde não fosse reconhecida, não haveria solução para os problemas; sempre haverá pessoas dispostas a se mobilizar para isso, qualquer que seja o fundamento moral, porque existe um senso altruísta inerente ao ser humano. No entanto, o reconhecimento de um direito permite alterar a atual distribuição de poder e de status - "The key point is that rights concern the distribution of power and status". ${ }^{(22)} \mathrm{O}$ reconhecimento de um direito cria um instrumento por meio do qual as pessoas são individualmente capazes de influenciar e gerar decisões em favor de si, de modo a alterar a atual

\footnotetext{
${ }^{19}$ WOLFF, Jonathan. op. cit., p. 9.

${ }^{20}$ Id. Ibid., p.16-17.

${ }^{21}$ Id. Ibid., p.14.

${ }^{22}$ Id. Ibid., p.15.
}

R. Dir. sanit., São Paulo, v. 14, n. 3, p. 299-306, nov. 2013/ fev. 2014 
distribuição de poder e status numa sociedade. Por outro lado, satisfazer-se com o altruísmo não gera um meio capaz de alterar a atual distribuição de poder e de status; pior do que isso: reforça a dependência daquele que a necessita ("developing world")(23) em relação àquele que a pode oferecer ("developed world")(24) e perpetua a atual distribuição. Fica claro que a solução altruísta limita substancialmente sua efetividade. Além disso, uma mera solução altruísta atinge apenas problemas urgentes, não amplos, constantes, crônicos, de modo que, em última instância, essa solução não gera efeitos sobre a base dos problemas (a distribuição desigual de poder).

O autor argumenta que deve ser reconhecido o status jurídico da saúde, pois altera a distribuição de poder e de status. Isso o aproxima da corrente comunitarista (especificamente, da teoria de Michael Walzer), de acordo com a qual poder e status são multiplicados por meio do "monopólio"(25) do "bem social", cujo valor de troca permite àquele (ou ao grupo) que o detém trocá-lo por todos os outros bens sociais existentes na sociedade ("bem predominante") ${ }^{(26)}$ - afinal, poder e status seriam, em si, bens sociais. Evidentemente, esse processo depende de que todos os indivíduos compartilhem significados, juízos de valoração sobre os bens sociais. Também os direitos, como instrumentos capazes de alterar a distribuição de poder e de status, só seriam capazes disso numa sociedade em que todos os indivíduos compartilhassem significados, juízos de valoração sobre os bens sociais. Em nível global, seria necessário um exercício interpretativo no sentido de demonstrar que, de fato, existem valores compartilhados por todo e qualquer ser humano - e uma interpretação coerente com essa teoria não admitiria concluir que a liberdade individual é um valor comum. Isso porque, se assim fosse, implicaria dizer que apenas direitos fundamentados na liberdade são capazes de alterar a distribuição de poder na sociedade, ao passo que, realmente, o que isso geraria seria uma tendência no sentido da atomização, da fragmentação da sociedade.

\section{Crítica}

Embora o autor tenha explicado a importância do reconhecimento da saúde de forma a que permitisse a distribuição de poder e de status entre os países, endossando a teoria comunitarista e, inclusive, defendendo sua aplicabilidade em nível global, ele, curiosamente, quando considera a existência de diversos fundamentos morais que justificam os direitos humanos, em vez de iniciar um exercício interpretativo no sentido de identificar (ou descobrir) fundamentos, valores comuns (como esperado para corroborar a possibilidade da distribuição de poder e de status na sociedade global), acaba reconhecendo que os diversos

\footnotetext{
${ }^{23}$ WOLFF, Jonathan. op. cit., p. 91.

${ }^{24}$ Id., loc. cit.

${ }^{25}$ WALZER, Michael. Esferas da justiça: uma defesa do pluralismo e da igualdade. São Paulo: Martins Fontes, 2003. p. 11-20.

${ }^{26}$ Id. Ibid.,
} 
fundamentos morais são um fato inexorável (de maneira semelhante ao "fato do pluralismo razoável"(27) de John Rawls):

What, after all, are the foundations of human rights? What are the arguments for believing in them? In fact there are many arguments: that they are based on our common humanity, or on human dingnity, or on our nature as human agents, or on our basic goods, or even on God's will. Our problem is not that there are no foundations for human rights, but that there are too many. Which is the correct account of the foundations? We are unlikely to be able to find a conclusive, universally convincing, single argument or account. ${ }^{(28)}$

Tendo reconhecido que o pluralismo de fundamentos morais é um fato, o autor, em seguida, acrescenta que, apesar disso, geralmente existe consenso sobre o que deve ser reconhecido como direito humano: "For what is as true now as sixty years ago is that there is much greater agreement on the broad list of human rights than there is on their moral foundations". ${ }^{(29)} \mathrm{E}$ explica de que forma essa situação é possível com base na ideia rawlsiana de "consenso sobreposto" ("overlapping consensus"):(30)

To use terminology from political philosopher John Rawls, the Universal Declaration of Human Rights may be seen as analogous to Rawls's idea of na 'overlapping consensus', in which each person can endorse a political doctrine for his or her own moral reasons. (...). This, perhaps, explains the appeal of human rights doctrine within a broadly liberal framework, in that it does not pressupose that everyone accepts the same basic moral theory. ${ }^{(31)}$

É preciso dizer, contudo, que tanto o entendimento apresentado inicialmente quanto este último parecem incompatíveis. É que postular o reconhecimento da saúde com base em que ela permitiria uma distribuição do poder e do status pressupõe, como esclarecido, um compartilhamento de valores; por outro lado, ao buscar uma explicação sobre como existe consenso em torno dos direitos humanos, mesmo com diversos fundamentos morais, o autor reconhece que esse pluralismo é um fato, mais do que isso, inquestionável -

at the level of detail there is no consensus. Probably the right response at this point is simply to acknowledge the limits of philosophical argument, and allow the resulting disputes to be resolved through the development of democratic politics and legal doctrine. ${ }^{(32)}$

\footnotetext{
${ }^{27}$ RAWLS, John. O liberalismo político. São Paulo: WMF Martins Fontes, 2011. p. XL-XLII.

${ }^{28}$ WOLFF, Jonathan. op. cit. p. 19.

${ }^{29}$ Id. Ibid. p. 19-20.

${ }^{30}$ RAWLS, John. op. cit. p. 157.

${ }^{31}$ WOLFF, Jonathan. op. cit. p. 20.

${ }^{32}$ Id., loc. cit.
} 
Além disso, se se abandonar totalmente o primeiro entendimento em favor de um total alinhamento com o segundo (reconhecendo que os diversos fundamentos morais são um fato), não parece certo que a teoria rawlsiana seja adequada para explicar como, mesmo com os diversos fundamentos morais, existe consenso sobre o que deve ser reconhecido como direito humano. No entanto, não é possível formular essa ideia nos limites deste trabalho.

\section{Considerações finais}

Não se nega a importância do reconhecimento da saúde como um direito humano. O autor é bastante convincente sobre isso, ao descrever como os direitos humanos contribuíram para lidar com os problemas relacionados à AIDS. Segundo ele, direitos humanos, pouco mencionados até então, serviram como instrumento para legitimar e potencializar as mobilizações contra a violação de deveres morais relacionados à AIDS. Por outro lado, o próprio sentido dos direitos humanos foi, em grande medida, determinado por ocasião dessas mobilizações.

Não obstante, não se pode ignorar a necessidade de buscar uma explicação teórica que justifique satisfatoriamente a doutrina dos direitos humanos, de modo, inclusive, a contribuir para a solução dos diversos problemas práticos que persistem até hoje.

\section{Referências}

UNIVERSITY COLLEGE LONDON. Professor Jonathan Wolff. Homepages. Disponível em: <http://www.homepages.ucl.ac.uk/ uctyjow/>. Acesso em: 15 dez. 2013.

UNIVERSITY COLLEGE LONDON. Professor Jonathan Wolff. UCL. Disponível em: <http://www.ucl.ac.uk/cpjh/people/JoW>. Acesso em: 15 dez. 2013.

WOLFF, Jonathan. The human right to health. 1. ed. New York: W. W. Norton \& Company, 2013. (Norton Global Ethics Series)

RAWLS, John. Uma teoria da justiça. São Paulo: Martins Fontes, 2008.

Fontes, 2011.

O liberalismo político. Edição ampliada. São Paulo: WMF Martins

WALZER, Michael. Esferas da justiça: uma defesa do pluralismo e da igualdade. São Paulo: Martins Fontes, 2003.

WALZER, Michael. A crítica comunitarista do liberalismo in Política e paixão: rumo a um liberalismo mais igualitário. São Paulo: WMF Martins Fontes, 2008. 